\title{
Long-term incubation of lake water enables genomic sampling of consortia involving Planctomycetes and Candidate Phyla Radiation bacteria
}

\author{
Alexander L. Jaffe ${ }^{1}{ }^{\dagger}$, Maxime Fuster ${ }^{2}{ }^{\dagger}$, Marie C. Schoelmerich ${ }^{3}$, Lin-Xing Chen ${ }^{4}$, Jonathan \\ Colombet $^{2}$, Hermine Billard ${ }^{2}$, Télesphore Sime-Ngando ${ }^{2}$, Jillian F. Banfield ${ }^{3,4,5,6^{*}}$ \\ ${ }^{1}$ Department of Plant and Microbial Biology, University of California, Berkeley, CA \\ ${ }^{2}$ Laboratoire Microorganismes: Génome et Environnement (LMGE), UMR CNRS 6023, Université \\ Clermont-Auvergne, F-63000 Clermont-Ferrand, France \\ ${ }^{3}$ Innovative Genomics Institute, University of California, Berkeley, CA \\ ${ }^{4}$ Department of Earth and Planetary Science, University of California, Berkeley, CA \\ ${ }^{5}$ Department of Environmental Science, Policy, and Management, University of California, Berkeley, CA \\ ${ }^{6}$ Chan Zuckerberg Biohub, San Francisco, CA \\ ${ }^{\dagger}$ These authors contributed equally. \\ ${ }^{*}$ Corresponding author email: jbanfield@berkeley.edu
}

\begin{abstract}
Microbial communities in lakes can profoundly impact biogeochemical processes through their individual activities and collective interactions. However, the complexity of these communities poses challenges, particularly for studying rare members. Laboratory enrichments can select for subsystems of interacting organisms and enable genome recovery for enriched populations. Here, a reactor inoculated with water from Lake Fargette, France, and maintained under dark conditions at $4^{\circ} \mathrm{C}$ for 31 months enriched for diverse Planctomycetes and Candidate Phyla Radiation (CPR) bacteria. We reconstructed draft genomes and predicted metabolic traits for 12 diverse Planctomycetes and 9 CPR bacteria, some of which are likely representatives of undescribed families or genera. One CPR genome representing the little-studied lineage Peribacter (1.239 Mbp) was curated to completion, and unexpectedly, encodes the full gluconeogenesis pathway. Metatranscriptomic data indicate that some Planctomycetes and CPR bacteria were active under the culture conditions. We also reconstructed genomes and obtained transmission electron microscope images for numerous phages, including one with a $>300 \mathrm{kbp}$ genome and several predicted to infect Planctomycetes. Together, our analyses suggest that freshwater Planctomycetes may act as hubs for interaction networks that include symbiotic CPR bacteria and phages.
\end{abstract}

\section{INTRODUCTION}

Freshwater lakes host diverse microbial communities that likely control ecosystem biogeochemistry [1, 2]. Here, we established a laboratory culture based on an inoculum from Lake Fargette, France, and probed enriched populations using a combination of metagenomics, metatranscriptomics, and microscopy. This site was originally chosen for a parallel study of enigmatic 'aster-like nanoparticles' (ALNs) [3, 4]. We recovered draft genomes for abundant and transcriptionally active Planctomycetes, as well as CPR bacteria, phages, and eukaryotic 
bioRxiv preprint doi: https://doi.org/10.1101/2021.09.01.458585; this version posted September 1, 2021. The copyright holder for this preprint (which was not certified by peer review) is the author/funder, who has granted bioRxiv a license to display the preprint in perpetuity. It is made available under aCC-BY-NC-ND 4.0 International license.

viruses. Overall, we provide clues to interactions among microbial groups in a lake ecosystem that were linked by co-enrichment.

a.
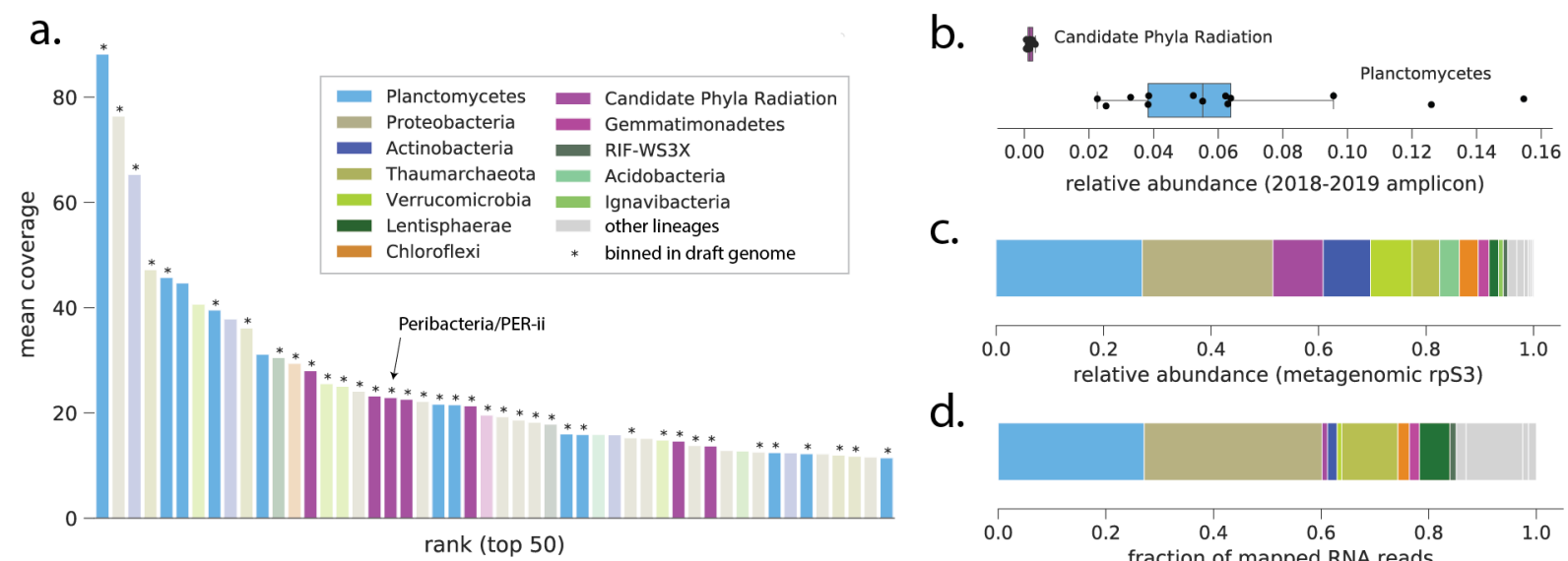

C.

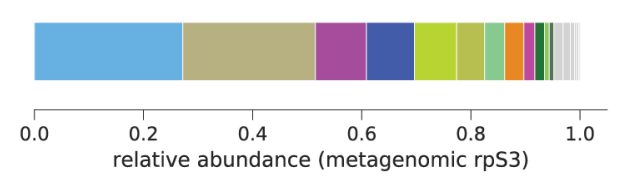

d.

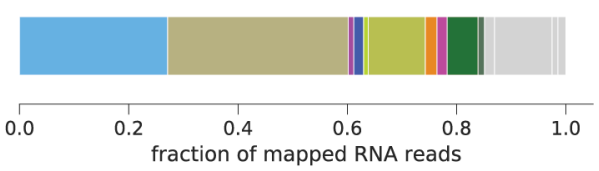

e.

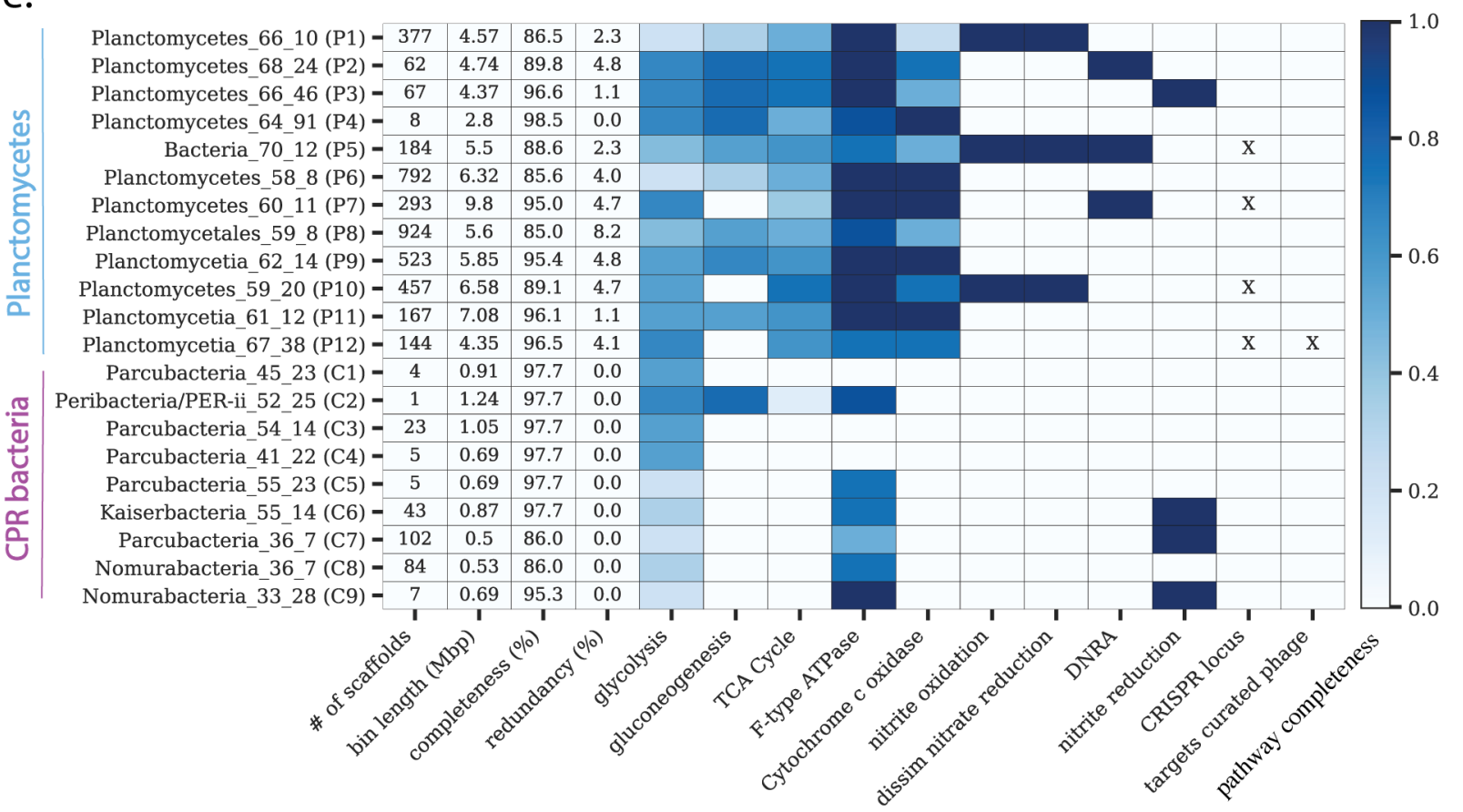

Fig. 1: Long-term incubation enriched for members of the Planctomycetes and CPR bacteria. a) Rank abundance curve based on ribosomal protein S3 (rps3) coverage for organisms recovered at the end of incubation. Asterisks indicate marker genes that were binned into genomes. b) Relative abundance of CPR bacteria and Planctomycetes during monthly sampling of Lake Fargette. Each point represents the relative abundance of CPR bacteria or Planctomycetes in a given month. c) Overall community composition at the end of incubation, based on cumulative marker gene coverage. Panel $\mathbf{d}$ displays the fraction of RNA reads from the end of incubation that could be mapped to genomes. e) Sequence characteristics, metabolic predictions, and CRISPR-Cas loci information for genomes affiliated with the Planctomycetes and CPR bacteria. Cells with color fill indicate the fraction of key genes for each pathway (as defined by KEGGDecoder) that are present. "X"s indicates genomes with CRISPR loci, and if those loci contained spacers targeting at least one curated phage genome from the sample. 


\section{RESULTS}

\section{Community composition and genome reconstruction}

Concentrate from the $0.2 \mu \mathrm{m}-25 \mu \mathrm{m}$ size fraction of the highly eutrophic Lake Fargette, France, was incubated at $4^{\circ} \mathrm{C}$ in the dark with filtered and sterilized lake water ( $\left.<20 \mathrm{KDa}\right)$. After 31 months, DNA and RNA were extracted for metagenomic and transcriptomic analyses. DNA reads were assembled and the contigs profiled to identify ribosomal protein S3 (rpS3). Profiling using the predicted rpS3 protein sequences revealed that the enrichment was bacterially-dominated, although several members of the Thaumarchaeota were present (Fig. 1a, Table S1). The most abundant organisms overall were Planctomycetes ( $27 \%$ of overall rpS3 coverage), including the most abundant singular organism (Fig. 1, Table S1). CPR bacteria were 5 of the top 25 most abundant organism groups (overall, 9\%; Fig. 1, Table S1). When compared to baseline abundances in Lake Fargette, these results indicate enrichment of these groups, particularly CPR bacteria, which were barely detectable in the lake (Fig. 1b, Table S2).

Where possible, scaffolds were assigned to genome bins that ranged in quality from draft to near-complete. The 48 genomes captured most of the phylogenetic diversity (38 of 50 most abundant rpS3 genes; Fig. 1a, Table S1/S3). Twelve genomes represent phylogenetically diverse Planctomycetes, including several from the large Planctomycetes and Phycisphaerae classes (Fig. S1, Table S3). Metabolic reconstructions suggested that the Planctomycetes are primarily heterotrophs with the potential to oxidize/reduce nitrite in some cases (Fig 1e). We also recovered 9 genomes of CPR bacteria, all but one of which came from lineages within the Parcubacteria (Fig. S1). The Parcubacteria genomes encoded minimal metabolic capacities consistent with symbiotic lifestyles [5]. However, several encoded a phylogenetically distinct nirK gene that may play a role in denitrification or energy conservation [6] (Fig. 1e).

One of the draft CPR genomes was for a member of the undersampled CPR lineage Peribacteria. We manually curated the original bin of 6 fragments into a single fragment that was circularized using a small, unbinned contig (Fig. S2, Materials and Methods). The newly reported 1,239,242 bp genome shares $~ 89 \%$ similarity in its 16 S rRNA gene and is largely syntenous with a closely-related Peribacteria genome from Rifle, Colorado [7], supporting the accuracy of both assemblies (Fig. S3). Unlike the Rifle genomes, this Peribacter likely cannot synthesize purines de novo. Other notable differences include the presence of vacuolar-type $\mathrm{H}+/ \mathrm{Na}+-$ transporting ATPase complex and the lack of genes for biosynthesis of mevalonate. Based on biosynthetic deficiencies, we conclude that this bacterium probably relies on other organisms for many building blocks, but less so than the Parcubacteria. Supporting this idea, we observed that the Peribacter genome encodes all gluconeogenesis enzymes, including fructose-1,6-bisphosphatase I, which was not found in the Parcubacteria genomes (Fig. 1e).

\section{Phage and virus genomes}

We reconstructed 12 phage sequences and 5 phage-like sequences, including three circularized genomes exceeding $100 \mathrm{kbp}$ and two incomplete phage/phage-like fragments $>300 \mathrm{kbp}$ (Table 
S4). Phylogenetic analyses of encoded terminase and capsid proteins suggested that the phages likely fall within the Caudovirales, which is known to include numerous tailed phages with large capsids [8]. Additionally, we used phage gene content and analyses of bacterial CRISPR loci to infer that phages infect Planctomycetes, Proteobacteria, and Bacteroidetes (Fig. 2, Table S4, Materials and Methods).

We also reconstructed large fragments of 17 eukaryotic viruses. Based on the phylogenetic placement of the major capsid protein and homologs of the Poxvirus Late Transcription Factor VLTF3, some viruses belonged to Iridoviridae (Betairidoviridae), Extended Mimiviridae, Phycodnaviridae, and Pitho-like viruses (Fig. S4-5, Table S4). Other viruses could only be classified at the superclade level, including those within a potentially novel Phycodnaviridae, Asfarviridae, Megavirales (PAM) clade, or did not contain either marker protein (Fig. S4-5).
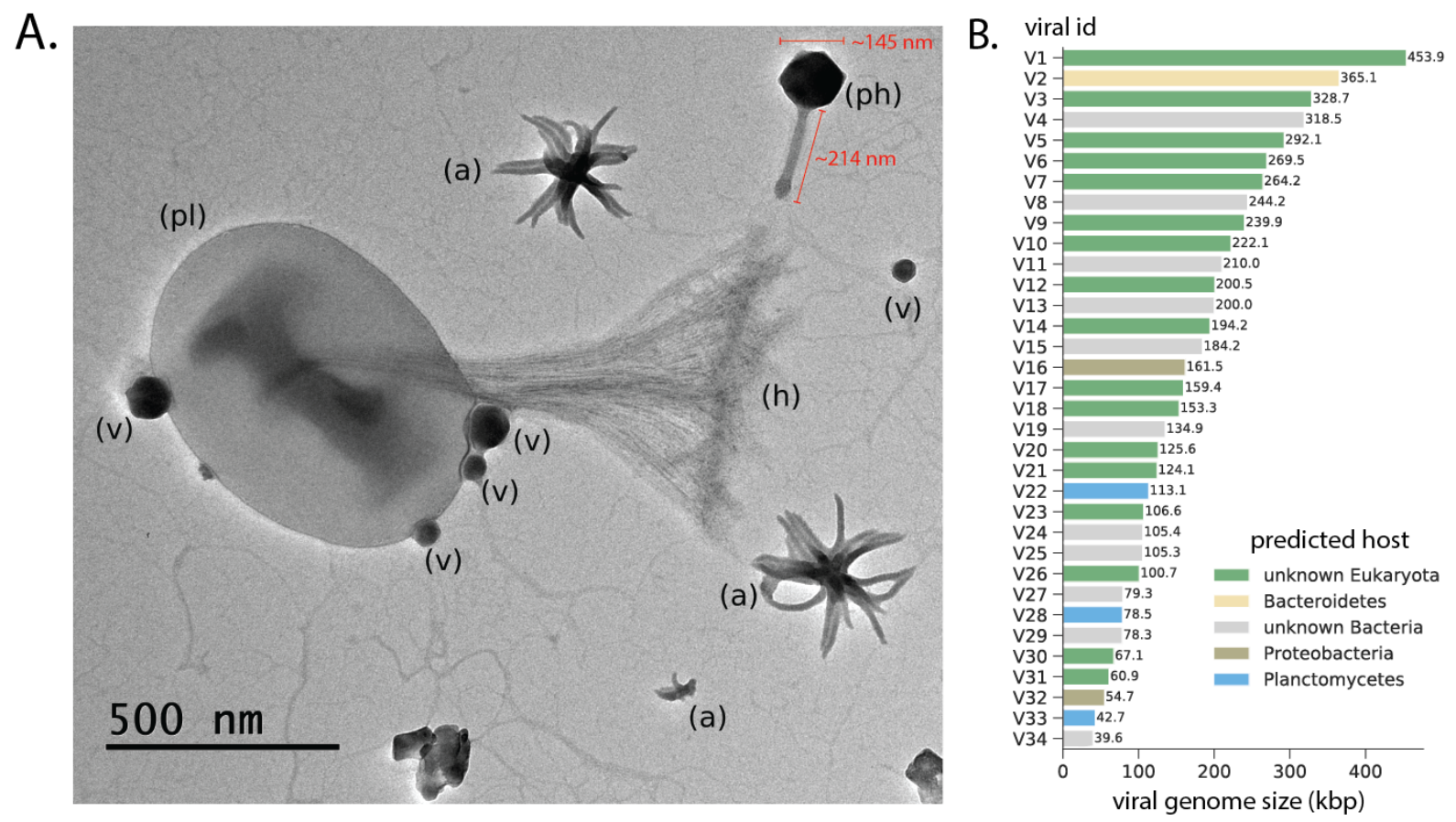

Fig. 2: TEM imaging and viral genomics in the enrichment culture. A. Featured in this image is a cell inferred to be a Planctomycetes ( $\mathrm{pl}$ ) with a characteristic stalk and holdfast $(\mathrm{h})$. Attached to the cell are four phage particles ( $v$ ) (two different sizes, thus likely different phages). Also visible is one large tailed jumbo phage (ph) that is $145 \mathrm{~nm}$ in diameter with a $214 \mathrm{~nm}$ tail, as well as several aster-like nanoparticles (ALNs) (a). B. Genome sizes and predicted hosts for phages and eukaryotic viruses.

\section{Imaging of incubation community}

We imaged a diversity of cellular, cellular-like, and non-cellular particles using transmission electron microscopy (TEM) (Fig. 2, Fig. S6, Materials and Methods). Based on the presence of the extracellular 'holdfast' [9, 10], we infer that the cell imaged in Fig. 2 is likely a member of the Planctomycetes, with at least two attached distinct types of virus-like particles (VLPs). This finding is consistent with the high abundance of Planctomycetes in the enrichment as well as multiple phages predicted to infect them ( 18-100x coverage). 
We imaged numerous tailed phages (Fig. S6), including one with a $145 \mathrm{~nm}$ diameter capsid and $214 \mathrm{~nm}$ tail ("ph", Fig. 2). This large capsid size is consistent with those of jumbo phages with genomes in the $300 \mathrm{kbp}$ range [8], two of which were reconstructed here (Table S4). Despite these observations, TEM image counts suggested that the overall proportion of tailed phages decreased from $26 \%$ to less than $1 \%$ of imaged objects over the course of the incubation (Table S5). Conversely, the proportion of ALNs increased substantially during the incubation, from $26 \%$ to $36 \%$ of imaged objects during the same period (Table S5).

\section{Transcriptomics of incubated community}

Read mapping from a metatranscriptome collected with the metagenome suggested that Planctomycetes, and to a lesser extent, CPR bacteria, were active under the culture conditions, with Planctomycetes accounting for about $27 \%$ of RNA reads mapping to the non-redundant set of genomes (Fig 1d, Table S3). Interestingly, we detected very little transcription of genes from phage or eukaryotic viruses, suggesting that they were not actively replicating in the incubated community (Table S4). The lack of eukaryotes in the enrichment suggests that some of these particles may have derived from the inoculum and persisted for over two years.

\section{DISCUSSION}

Planctomycetes are globally distributed across freshwater ecosystems where they are thought to play important roles in nitrogen and carbon cycling [11, 12]. Our analyses both expand genomic sampling for these organisms and suggest their interactions with other microbial groups, potentially including CPR bacteria. Although we cannot establish that the CPR bacteria [13] are symbionts of Planctomycetes, their co-enrichment suggests a possible association. Thus, the findings could guide future co-isolation of the CPR bacteria of the Parcubacteria and Peribacteria lineages, for which very little is currently known about host relationships [14].

It is also intriguing to find Planctomycetes and CPR bacteria co-enriched with ALNs. ALNs are enigmatic, organic femtoplankton entities that exhibit bloom-like behavior in various freshwater and coastal environments [3, 4]. Their defined morphologies suggest that they develop under genetic control; however, the genetic system responsible for producing ALNs remains unknown. Our co-enrichment data suggest ALNs may interact with or derive from co-occurring bacteria.

Overall, our results suggest that Planctomycetes might act as 'hubs of interactions' in subsystems within certain freshwater ecosystems.

\section{MATERIALS AND METHODS:}

In vitro incubation, selection, and sequencing of microbial communities associated with ALNs

Lake Fargette is an artificial and highly eutrophic freshwater lake (surface area 1.2 ha, maximum depth $2.5 \mathrm{~m}$ ) near Neuville in the French Massif Central (4544'24'N; 3²7'39'E; 465 
m altitude). Lake water was collected on March 15, 2017 when the highest density of ALNs was recorded in $2017\left(9.0 \pm 0.5 \times 10^{7} \mathrm{ALNs} \cdot \mathrm{mL}^{-1}\right)$. Information about this sample is provided in Colombet et al. 2019 [3]. Within two hours after sampling, $100 \mathrm{~L}$ of raw lake water was filtered through a $25-\mu m$-pore-size nylon mesh. Microbial communities were enriched by tangential-flow ultrafiltration using a Kross-Flow system (Spectrum, Breda, The Netherlands) equipped with a $0.2-\mu \mathrm{m}$ cut-off cartridge. Aliquots $(600 \mathrm{~mL})$ of this concentrated $0.2 \mu \mathrm{m}-25 \mu \mathrm{m}$ fraction were sequentially centrifuged at $8,000 \mathrm{~g}, 10,000 \mathrm{~g}$ (pellets discarded) then $12,000 \mathrm{~g}$ for 20 minutes each time at $14^{\circ} \mathrm{C}$. Microbial communities contained in the final supernatant were cultivated for an initial period of 6 months at $4^{\circ} \mathrm{C}$ in the dark. The physico-chemical parameters of the starting sample are listed in Table S6.

After 6 months, the selected microbial community was enriched by centrifugation at $6000 \mathrm{~g}$ for 20 minutes at $14^{\circ} \mathrm{C}$ and the pellet (in liquid phase) incubated 25 more months at $4^{\circ} \mathrm{C}$ in the dark. At the end of the incubation, the medium was sonicated using Elmasonic S30 (Elma, Germany) and microbial communities were pelleted by centrifugation at $6000 \mathrm{~g}$ for 20 minutes at $14^{\circ} \mathrm{C}$. The pellet suspended in distilled deionized water was used for nucleic acid extraction and amplification.

DNA and RNA extractions were performed using the RNA x DNA from soil kit (740143, Macherey-Nagel, Germany). The RNA sample was treated with Turbo DNA-free Kit (Invitrogen, Massachusetts, USA). The concentration of the samples was checked using a Qubit fluorometer (Invitrogen, Massachusetts, USA).

\section{TEM imaging of enrichment communities}

Microbial communities were imaged at T0 and T31 to visualize their evolution, using a JEOL JEM 2100-Plus microscope (JEOL, Akishima, Tokyo, Japan) operating at $80 \mathrm{kV}$ and 40,000x magnification. For each sample, 10 images were randomly captured in order to have a significant representation of microbial communities. We then defined the percentage of different observed phenotypes as a proportion of the total observed communities (Table S5).

\section{Monthly microbial community profiling at Lake Fargette}

Samples were collected every month for 1 year (10/2018 to 09/2019) from the depth interval of $0-40 \mathrm{~cm}$ of lake Fargette. For each sample, microbial communities were collected on a $0.2 \mu \mathrm{m}$ (Millipore) polycarbonate filter (until saturation, pressure $<25 \mathrm{kPa}$ ) and stored at $-20^{\circ} \mathrm{C}$ until DNA extraction. The filters were covered with a lysing buffer (lysozyme $2 \mathrm{mg} \cdot \mathrm{ml}-1 \mathrm{SDS} 0.5 \%$, Proteinase $\mathrm{K} 100 \mu \mathrm{g} \cdot \mathrm{mL}-1$ and RNase A $8.33 \mu \mathrm{g} \cdot \mathrm{mL}-1$ in TE buffer $\mathrm{pH} 8$ at $37^{\circ} \mathrm{C}$ for 90 minutes. A CTAB $10 \% / \mathrm{NaCl} 5 \mathrm{M}$ solution was added, and the samples were incubated at $65^{\circ} \mathrm{C}$ for 30 minutes. The nucleic acids were extracted with phenol-chloroform-isoamyl alcohol (25:24:1) the aqueous phase containing the nucleic acids was recovered and purified by adding chloroform-isoamyl alcohol (24:1). The nucleic acids were then precipitated with a mixture of glycogen $5 \mathrm{mg} / \mathrm{mL}-1$, sodium acetate $3 \mathrm{M}$ and ethanol $100 \%$ overnight at $-20^{\circ} \mathrm{C}$. The DNA pellet was rinsed with ethanol (70\%), dried and dissolved in TE buffer. The DNA was purified with NucleoSpin $\circledast$ gDNA Clean-up (Macherey-Nagel). 
The amplification of the V4-V5 region of the bacterial small subunit rDNA was performed using the universal bacteria $515 \mathrm{~F}$ and $928 \mathrm{R}$ primers modified with barcodes. PCR was performed in a total volume of $50 \mu \mathrm{L}$ containing $1 \mathrm{x}$ final green reaction buffer, $2 \mathrm{mM}$ final $\mathrm{MgCl}_{2}, 0.2 \mathrm{mM}$ final dNTP, $100 \mu \mathrm{g} \cdot \mathrm{mL}^{-1}$ final BSA, $0.2 \mu \mathrm{M}$ final of primers, $0.025 \mathrm{U} . \mu \mathrm{L}^{-1}$ final PROMEGA GoTaq HotsStart G2 and $5 \mu \mathrm{L}$ of sample DNA.

To process the bacterial sequencing data (Illumina Miseq@ $2^{*} 250 \mathrm{bp}$ ), we used the FROGS pipeline [15]. After the clean-up procedure, sequences were assembled and clustered into OTUs with a similarity threshold of $95 \%$. The representative sequences of each OTU were affiliated by similarity using the SILVA_132_16s database.

\section{Metagenomic/metatranscriptomic sequencing, binning, and marker gene analysis}

Library preparation and sequencing of extracted DNA and RNA was performed at the Vincent J. Coates Genomics Sequencing Laboratory and Functional Genomics Laboratory at U.C. Berkeley. Extracted RNA was treated with the QiaSeq FastSelect kit to deplete rRNA. Samples were sequenced on a NovaSeq 6000 with 150 bp, paired-end reads. DNA and RNA were sequenced to a depth of $\sim 10 \mathrm{Gbp}$ and 25 million read pairs, respectively. Quality filtering and assembly of DNA reads, as well as annotation and binning of assembled scaffolds followed the workflow outlined in [16]. Genomes were classified at the phylum or class level based on the majority taxonomic affiliation of member contigs, and supplemented with BLAST searches of ribosomal proteins where necessary. Additionally, genome classification according to the GTDB scheme was assigned by running GTDB-tk with default parameters [17].

For the marker gene analysis (Fig. 1ac), we computed the mean coverage of scaffolds encoding a ribosomal protein S3 (rpS3) gene using bowtie2. Phylum-level taxonomic affiliation of these scaffolds was manually curated based on consensus gene-level taxonomy, binning information, and BLAST searches of protein sequence, where necessary.

\section{Quality filtering and genome curation for bacteria and archaea}

Genomic bins were profiled for single copy genes using CheckM. For CPR bacteria, a custom workflow was used with a set of 43 marker genes sensitive to lineage-specific losses of ribosomal proteins in this group. We filtered all bins to those $\geq 70 \%$ completeness and $\leq 10 \%$ contamination and removed redundancy in the set at the species level using dRep (95\% ANI) [18]. Retained genomic bins were manually 'polished' by removing contigs that were outliers in terms of GC content and coverage. Contigs with aberrant or ambiguous consensus taxonomy were manually expected if $\geq 5 \mathrm{kbp}$ or automatically removed if $<5 \mathrm{kbp}$.

For the Peribacteria genome (LakeFargette_0920_ALND_PER-ii_52_24), additional manual genome curation was performed. This involved fixing local scaffolding errors and extending the six original contigs using unplaced paired reads. The extended contigs were then joined based on end overlaps and paired read support. This resulted in two contigs, the larger of which was 
circularized and the other contained the 16S rRNA gene. Based on identification of a sequence within the larger contig that was also present at the end of the smaller contig, the larger contig was broken and joined to the smaller contig. This produced a single linear fragment. We used BLAST against the metagenome of the sample to identify a $4 \mathrm{kbp}$ fragment that perfectly aligned to the ends of the linear fragment, circularizing the genome. The circularization overlap was trimmed and paired read support was verified by visualization throughout the entire final genome. Finally, the start position was adjusted based on the cumulative GC skew (Fig. S2). Genes/proteins were then re-predicted for the curated genome using Prodigal (single) [19].

We selected one of a set of near-identical complete Peribacteria genomes (SAMN03842449) from a previous publication [7] for comparison to the newly curated genome. Full genomes and 16S rRNA sequences were aligned in Geneious (Mauve plugin and MAFFT aligner, respectively).

\section{Phylogenomic and gene content analyses for CPR bacteria and Planctomycetes}

For analyses of gene content, we focused on a subset of 21 genomes from the CPR bacteria and Planctomycetes. Gene/protein predictions were re-computed for this subset using Prodigal (single mode). Predicted protein sequences were annotated using kofamscan and resulting HMM hits were preliminarily filtered to those with $e<1 \times 10^{-6}$ for use in downstream phylogenetic and metabolic analyses.

First, proteins annotated as rpS3 (K02982) were extracted and combined with reference sequences drawn either from NCBI (Planctomycetes) or a previous publication [20] on CPR phylogeny as well as a balanced sampling of other bacterial phyla as an outgroup. Each sequence set was aligned using mafft (auto) [21] and the resulting alignment was trimmed using trimal (gt 0.1) [22]. Maximum-likelihood trees were inferred using iqtree (-m TEST -safe -st AA -bb 1500 -nt AUTO -ntmax 20) [23] and subsequently visualized/annotated using iTOL [24].

For analyses of metabolism, we stringently filtered the kofamscan results, requiring that protein hits attain a score equal to or greater than the model specific thresholds for each KO. Results were then passed to KEGGDecoder [25] for visualization. For CRISPR-Cas analyses, we employed a custom script that first identifies repeat regions on scaffolds using PILER-CR [26] with default parameters, then detects Cas protein using the TIGRFAM HMM database [27] using hmmsearch [28] within $10 \mathrm{kbp}$ (both upstream and downstream) of each identified repeat region. If at least one Cas protein was detected for a given repeat region, the spacer sequences from the adjacent CRISPR locus and also the paired reads and unplaced paired reads were extracted to identify spacer targets. Target identification was performed by blastn-short with an e-value threshold of $1 \times 10^{-3}$. Hits were further filtered to those in which the spacer and targeted sequence were aligned over $\geq 90 \%$ of the spacer length and with no more than 2 mismatches.

\section{Viral genome identification and analysis}


Viral genomes were identified through taxonomic profiling and identification of key viral structural proteins. While most recovered viral genomes were single-contig, in some cases multiple contigs of putative viral origin were binned together on the basis of GC content, coverage, and scaffold overlap. Viral genomes were tentatively classified as bacteriophage or eukaryotic viruses based on taxonomic profiling. Completeness and quality information for single-contig viruses was estimated using CheckV [29].

To establish phylogenetic affiliation of bacteriophages, we gathered curated sets of reference sequences for viral terminase and major capsid protein (MCP) from RefSeq. Reference sets were filtered to those sequences with sequence lengths $1 \pm 0.5$ times the median and subsequently de-replicated at $95 \%$ identity using usearch (-cluster_fast, -id 0.95) [30]. Proteins for the newly identified phage genomes were predicted using Prodigal (meta mode). Terminase and MCP sequences were identified using BLAST (-evalue 1e-20) against a database built from the reference set. Sequences were concatenated with those of the reference set and aligned using MAFFT (--reorder --auto). Alignments were trimmed using trimal (-gt 0.2) and maximum likelihood trees were inferred using IQtree (-m TEST -st AA, version 1.6.12). Trees were decorated using taxonomic information for RefSeq sequences and putative lineages were assigned to newly identified phage genomes based on tree placement.

Host prediction for phages was performed using a combination of CRISPR-Cas targeting (described above) and taxonomic profiling. Briefly, for phylogenetic profiling, phage proteins were compared against UniRef100 using a custom DIAMOND database (diamond blastp) [31]. Hits were filtered to those with $70 \%$ or greater coverage of the query sequence and an e-value less than or equal to $1 \times 10^{-10}$. We retrieved taxonomic affiliation for above-threshold hits and computed the percentage of genes on each phage with highest similarity to various bacterial lineages. If the bacterial lineage with the most hits among phage genes reached a percentage $\geq$ $3 x$ that of the next highest lineage, it was assigned as the putative phage host. This method was previously shown to be consistent with host prediction via CRISPR-Cas targeting [32].

To infer the taxonomy of the 17 putative viruses of eukaryotes, all proteins were profiled against the Pfam database. 17 major capsid proteins (PF16903|PF04451) from 11 eukaryotic virus bins and 8 VLTF3-like proteins (PF04947) from 8 bins served as markers. If there were multiple major capsid proteins per bin, the protein with the highest PFAM score was selected. The marker proteins were aligned with a reference dataset of capsid or VLTF3-like proteins from Nucleo-Cytoplasmic Large DNA Viruses [33] and 48 additional capsid proteins retrieved from NCBI (Extended Data File 1-2). The sequences were aligned, trimmed and the phylogenetic tree was constructed as described above. Finally, viruses were taxonomically classified based on phylogenetic placement.

\section{Transcriptome analysis}

RNA reads were subjected to the same quality filtering pipeline referenced for DNA reads above and were subsequently mapped to the quality-filtered, de-replicated set of bacterial and archaeal genomes using bowtie2. To remove remnant rRNA and other non-mRNAs, we 
computed per-gene mapped read counts using pysam and removed those $>100$ times the median non-zero per-gene read count for each genome. This process filtered about $\sim 60$ anomalously high-coverage ORFs, many of which corresponded to 16S rRNA or tRNA regions on scaffolds. Read counts were then aggregated by phylum-level lineage.

RNA reads were also mapped against the set of curated viral genomes described above. Mean coverage and coverage breadth were computed using CoverM (genome mode, --min-read-percent-identity 0.95 --min-covered-fraction 0).

\section{Data and software availability}

Read data and draft genomes from this study are available through NCBI at PRJNA757735. Genome accession information for the 48 bacterial and archaeal genomes is also listed in Table S3. Custom code for the described analyses are also available on GitHub (github.com/alexanderjaffe/aln-enrichment). All supplementary figures, tables, extended data files, and genomes (including phage/viral genomes) are also available through Zenodo (https://doi.org/10.5281/zenodo.5362897).

ACKNOWLEDGMENTS: We thank Alex Crits-Christoph, Adair Borges, Rohan Sachdeva, and Yue Clare Lou for informatics support and helpful discussions. We also thank Plateforme CYSTEM - UCA PARTNER (Clermont-Ferrand, France) for their technical support and expertise, the Innovative Genomics Institute and the Vincent J. Coates Genomics Sequencing Laboratory at UC Berkeley.

M.F., H.B., J.C., and T.S-N. performed sample collection, incubation experiments, and microscopy. A.L.J., L-X.C., M.C.S., and J.F.B. performed metagenomics and sequence analysis and J.F.B carried out the manual genome curation. All authors contributed to project design and manuscript writing.

COMPETING INTERESTS: J.F.B. is a founder of Metagenomi. The other authors declare no competing interests.

\section{FUNDING INFORMATION}

Funding was provided by the Innovative Genomics Institute at UC Berkeley, the Chan Zuckerberg Biohub, and a Moore Foundation Grant 71785. M.F. was supported by a PhD fellowship from the CPER 2015-2020 SYMBIOSE challenge program (French Ministry of Research, UCA, CNRS, INRA, Auvergne-Rhône-Alpes Region, FEDER). M.C.S. was supported by a DFG fellowship. This study is a contribution to the "C NO LIMIT" project funded by the Interdisciplinary Mission of the French National Center of Scientific Research (CNRS) Program $X$-life, 2018 edition. This research was also financed by the French government IDEX-ISITE initiative 16-IDEX-0001 (CAP 20-25).

\section{REFERENCES:}


1. Grossart H-P, Massana R, McMahon KD, Walsh DA. Linking metagenomics to aquatic microbial ecology and biogeochemical cycles. Limnol Oceanogr 2020; 65.

2. Tran PQ, Mclntyre PB, Kraemer BM, Vadeboncoeur Y, Kimirei IA, Tamatamah R, et al. Depth-discrete eco-genomics of Lake Tanganyika reveals roles of diverse microbes, including candidate phyla, in tropical freshwater nutrient cycling. bioRxiv . 2019. , 834861

3. Colombet J, Billard H, Viguès B, Balor S, Boulé C, Geay L, et al. Discovery of High Abundances of Aster-Like Nanoparticles in Pelagic Environments: Characterization and Dynamics. Front Microbiol 2019; 10: 2376.

4. Fuster M, Billard H, Mandart M, Steiger J, Sime-Ngando T, Colombet J. Trophic Conditions Influence Widespread Distribution of Aster-Like Nanoparticles Within Aquatic Environments. Microb Ecol 2020; 80: 741-745.

5. Castelle CJ, Brown CT, Anantharaman K, Probst AJ, Huang RH, Banfield JF. Biosynthetic capacity, metabolic variety and unusual biology in the CPR and DPANN radiations. Nat Rev Microbiol 2018; 16: 629-645.

6. Danczak RE, Johnston MD, Kenah C, Slattery M, Wrighton KC, Wilkins MJ. Members of the Candidate Phyla Radiation are functionally differentiated by carbon- and nitrogen-cycling capabilities. Microbiome 2017; 5: 112.

7. Anantharaman K, Brown CT, Burstein D, Castelle CJ, Probst AJ, Thomas BC, et al. Analysis of five complete genome sequences for members of the class Peribacteria in the recently recognized Peregrinibacteria bacterial phylum. PeerJ 2016; 4: e1607.

8. Yuan Y, Gao M. Jumbo Bacteriophages: An Overview. Front Microbiol 2017; 8: 403.

9. Boedeker C, Schüler M, Reintjes G, Jeske O, van Teeseling MCF, Jogler M, et al. Determining the bacterial cell biology of Planctomycetes. Nat Commun 2017; 8: 14853.

10. Jogler C, Glöckner FO, Kolter R. Characterization of Planctomyces limnophilus and development of genetic tools for its manipulation establish it as a model species for the phylum Planctomycetes. Appl Environ Microbiol 2011; 77: 5826-5829.

11. Wiegand $\mathrm{S}$, Jogler $\mathrm{M}$, Jogler $\mathrm{C}$. On the maverick Planctomycetes. FEMS Microbiol Rev 2018; 42: 739-760.

12. Andrei A-Ş, Salcher MM, Mehrshad M, Rychtecký P, Znachor P, Ghai R. Niche-directed evolution modulates genome architecture in freshwater Planctomycetes. ISME J 2019; 13 : 1056-1071.

13. He X, McLean JS, Edlund A, Yooseph S, Hall AP, Liu S-Y, et al. Cultivation of a human-associated TM7 phylotype reveals a reduced genome and epibiotic parasitic lifestyle. Proc Natl Acad Sci U S A 2015; 112: 244-249.

14. Jaffe AL, Thomas AD, He C, Keren R, Valentin-Alvarado LE, Munk P, et al. Patterns of Gene Content and Co-occurrence Constrain the Evolutionary Path toward Animal Association in Candidate Phyla Radiation Bacteria. MBio 2021; e0052121.

15. Escudié F, Auer L, Bernard M, Mariadassou M, Cauquil L, Vidal K, et al. FROGS: Find, Rapidly, OTUs with Galaxy Solution. Bioinformatics 2018; 34: 1287-1294.

16. He C, Keren R, Whittaker ML, Farag IF, Doudna JA, Cate JHD, et al. Genome-resolved metagenomics reveals site-specific diversity of episymbiotic CPR bacteria and DPANN archaea in groundwater ecosystems. Nat Microbiol 2021.

17. Chaumeil P-A, Mussig AJ, Hugenholtz P, Parks DH. GTDB-Tk: a toolkit to classify genomes with the Genome Taxonomy Database. Bioinformatics 2019.

18. Olm MR, Brown CT, Brooks B, Banfield JF. dRep: a tool for fast and accurate genomic comparisons that enables improved genome recovery from metagenomes through de-replication. ISME J 2017; 11: 2864-2868.

19. Hyatt D, Chen G-L, Locascio PF, Land ML, Larimer FW, Hauser LJ. Prodigal: prokaryotic gene recognition and translation initiation site identification. BMC Bioinformatics 2010; 11: 119.

20. Jaffe AL, Castelle CJ, Matheus Carnevali PB, Gribaldo S, Banfield JF. The rise of diversity 
in metabolic platforms across the Candidate Phyla Radiation. BMC Biology . 2020. , 18

21. Katoh K, Standley DM. MAFFT multiple sequence alignment software version 7: improvements in performance and usability. Mol Biol Evol 2013; 30: 772-780.

22. Capella-Gutiérrez S, Silla-Martínez JM, Gabaldón T. trimAl: a tool for automated alignment trimming in large-scale phylogenetic analyses. Bioinformatics 2009; 25: 1972-1973.

23. Nguyen L-T, Schmidt HA, von Haeseler A, Minh BQ. IQ-TREE: a fast and effective stochastic algorithm for estimating maximum-likelihood phylogenies. Mol Biol Evol 2015; 32: 268-274.

24. Letunic I, Bork P. Interactive tree of life (iTOL) v3: an online tool for the display and annotation of phylogenetic and other trees. Nucleic Acids Res 2016; 44: W242-5.

25. Graham ED, Heidelberg JF, Tully BJ. Potential for primary productivity in a globally-distributed bacterial phototroph. The ISME Journal . 2018. , 12: 1861-1866

26. Edgar RC. PILER-CR: fast and accurate identification of CRISPR repeats. BMC Bioinformatics 2007; 8: 18.

27. Haft DH, Selengut JD, White O. The TIGRFAMs database of protein families. Nucleic Acids Res 2003; 31: 371-373.

28. Johnson LS, Eddy SR, Portugaly E. Hidden Markov model speed heuristic and iterative HMM search procedure. BMC Bioinformatics 2010; 11: 431.

29. Nayfach S, Camargo AP, Schulz F, Eloe-Fadrosh E, Roux S, Kyrpides NC. CheckV assesses the quality and completeness of metagenome-assembled viral genomes. Nat Biotechnol 2021; 39: 578-585.

30. Edgar RC. Search and clustering orders of magnitude faster than BLAST. Bioinformatics 2010; 26: 2460-2461.

31. Buchfink B, Reuter K, Drost H-G. Sensitive protein alignments at tree-of-life scale using DIAMOND. Nat Methods 2021; 18: 366-368.

32. Al-Shayeb B, Sachdeva R, Chen L-X, Ward F, Munk P, Devoto A, et al. Clades of huge phages from across Earth's ecosystems. Nature 2020; 578: 425-431.

33. Guglielmini J, Woo AC, Krupovic M, Forterre P, Gaia M. Diversification of giant and large eukaryotic dsDNA viruses predated the origin of modern eukaryotes. Proc Natl Acad Sci $U$ S A 2019; 116: 19585-19592. 